of $\mathrm{CaPv}$ in diamonds that formed in the deep mantle supports Gréaux and colleagues' hypothesis. However, inclusions of a hydrous mineral ${ }^{13}$ and of pressurized ice ${ }^{14}$ in two other diamonds point to the presence of watercontaining fluids at similar depths, in support of the alternative hypothesis.

Global-scale geodynamic simulations ${ }^{9}$ indicate that oceanic crust descending into the mantle accumulates to form a layer that is enriched in basaltic rocks, centred at a depth of around $600 \mathrm{~km}$ - that is, at shallower depths than would be inferred from experimentally derived rock densities alone $e^{4,7,8}$. Gréaux and colleagues' results show that, in basalt, the velocities of the two types of seismic wave (known as shear (S) waves and compressional (P) waves) remain lower than global average seismic velocities at that depth, although the reduction in velocity is less than the reduction that occurs at depths greater than $660 \mathrm{~km}$. It is known that P waves are converted to $S$ waves at depths of around $600 \mathrm{~km}$ by globally distributed zones that have belowaverage $S$-wave velocities ${ }^{15}$. The idea that a layer of basaltic rocks scatters seismic waves at around $600 \mathrm{~km}$ depth could reconcile the seismic observations with the geodynamic predictions and with Gréaux and colleagues' models derived from the measured physical properties of minerals.

Further seismological studies are necessary to map zones that have low seismic velocities through a range of depths, and to better constrain their characteristics - for example, to measure differences in the velocities of $\mathrm{P}$ and $\mathrm{S}$ waves relative to the surrounding mantle. Measurements of sound-wave velocities in single crystals of $\mathrm{CaPv}$ (rather than in polycrystalline samples, as studied by Gréaux et al.) would also reveal how such velocities depend on the direction of passage through the crystal lattice. The effect of the crystal lattice might give rise to an observable direction dependence of seismic-wave velocities in the mantle. Devising models that combine seismological data with constraints derived from geodynamic simulations and data for the physical properties of minerals will aid the search for recycled oceanic crust in Earth's mantle.

Johannes Buchen is in the Seismological Laboratory, California Institute of Technology, Pasadena, California 91125, USA. e-mail:jobuchen@caltech.edu

1. Johnson, T. E., Brown, M., Kaus, B. J. P. \& VanTongeren, J. A. Nature Geosci. 7, 47-52 (2014).

2. Gréaux, S. et al. Nature 565, 218-221 (2019).

3. Irifune, T., Sekine, T., Ringwood, A. E. \& Hibberson, W. O. Earth Planet. Sci. Lett. 77, 245-256 (1986).

4. Irifune, T. \& Ringwood, A. E. Earth Planet. Sci. Lett. 117, 101-110 (1993).

5. Stixrude, L., Lithgow-Bertelloni, C., Kiefer, B. \& Fumagalli, P. Phys. Rev. B 75, 024108 (2007).

6. Kawai, K. \& Tsuchiya, T. Geophys. Res. Lett. 42 , 2718-2726 (2015).

7. Kesson, S. E., Fitz Gerald, J. D. \& Shelley, J. M. G. Nature 372, 767-769 (1994).
8. Hirose, K., Fei, Y., Ma, Y. \& Mao, H.-K. Nature 397 , 53-56 (1999).

9. Ballmer, M. D., Schmerr, N. C., Nakagawa, T. \& Ritsema, J. Sci. Adv. 1, e1500815 (2015).

10.Schmandt, B., Jacobsen, S. D., Becker, T. W., Liu, Z. \& Dueker, K. G. Science 344, 1265-1268 (2014). 11.Liu, Z., Park, J. \& Karato, S. Geophys. Res. Lett. 43,
2480-2487 (2016)

12. Nestola, F. et al. Nature 555, 237-241 (2018).

13.Pearson, D. G. et al. Nature 507, 221-224 (2014),

14. Tschauner, O. et al. Science 359, 1136-1139 (2018).

15.Shen, X., Yuan, X. \& Li, X. Geophys. Res. Lett. 41 , 836-842 (2014).

\title{
Immune cells track hard-to-target tumours
}

Clinical trials reveal that personalized vaccines can boost immune-cell responses to brain tumours that don't usually respond to immunotherapy. The findings also point to how to improve such treatments. SEE LETTERS P.234 \& P.240

\section{NEEHA ZAIDI \& ELIZABETH M. JAFFEE}

$\int \mathrm{n}$ nstructing the immune system to recognize and kill tumours, an approach termed cancer immunotherapy, has transformed the clinical treatment of certain types of malignancy. Prominent among these therapies are immune-checkpoint inhibitors, which block the action of proteins that dampen immune-cell responses against tumours. For example, antibodies can be used to interfere with the inhibitory protein PD-1, which is present on T cells, a type of immune cell that attacks tumours. Immune-checkpoint inhibitors have been most successfully used to treat cancers, such as melanomas, that are well infiltrated by $\mathrm{T}$ cells and have a large number of genetic mutations ${ }^{1,2}$. A subset of these mutations might generate neoantigens - altered protein sequences that are uniquely produced in cancer cells and are recognized as foreign by the immune system ${ }^{3}$.

Most cancers, however, including brain tumours called glioblastomas, do not respond to immune-checkpoint therapy. These nonresponsive tumours typically have a low level of mutations and express few neoantigens; that is, they have an immunosuppressively 'cold' tumour microenvironment. They therefore fail to attract $T$ cells that can infiltrate the tumour. Finding ways to boost an immune response to such tumours is an ongoing challenge. Keskin et al. ${ }^{4}$ (page 234) and Hilf et al. ${ }^{5}$ (page 240) report their progress in this area, and present the results of separate phase I clinical trials in which people with glioblastoma were treated with a personalized vaccine containing neoantigens that were specific to the individual's own tumour (Fig. 1).

Keskin et al. gave eight people who had undergone surgery to remove their tumour, and had received radiotherapy, a vaccine containing up to 20 protein fragments corresponding to neoantigens expressed in the person's tumour. These neoantigens were chosen by analysing the tumour material removed during surgery. Analysis of blood samples from the people who had been vaccinated then revealed whether types of $\mathrm{T}$ cell called helper $\mathrm{CD}^{+} \mathrm{T}$ cells and killer $\mathrm{CD} 8^{+}$ $\mathrm{T}$ cells were responding to these neoantigens.

The authors found that the vaccine failed to elicit a robust $\mathrm{T}$-cell response in the participants who had also been treated with the drug dexamethasone, which is a potent steroid immunosuppressant used to decrease swelling around the brain. The two people who had not received dexamethasone exhibited a neoantigen-specific T-cell response consisting of predominantly $\mathrm{CD} 4^{+} \mathrm{T}$ cells as well as $\mathrm{CD}^{+} \mathrm{T}$ cells. Notably, these neoantigen-specific $\mathrm{T}$ cells secreted proteins called cytokines, which are involved in killing tumour cells. These two people had T cells that expressed surface proteins known to be a hallmark of $\mathrm{T}$ cells that have acquired memory status - a characteristic that enables a faster and more robust immune response to a particular neoantigen if it subsequently re-emerges. However, all the vaccinated patients, even the two who responded, ultimately died of the cancer.

One key finding of this study relates to the $\mathrm{T}$ cells present in surgically removed samples of recurring tumour growth in one of the responders' tumours. In this case, the neoantigen-specific $T$ cells in the tumour expressed proteins indicating that the cells had entered a dysfunctional state termed exhaustion. This state corresponds to a decreased ability to recognize and kill cancer cells. Exhausted T cells can sometimes be reinvigorated using immune-checkpoint inhibitors.

Hilf et al. tested a strategy that used two types of vaccine. One of these, similar to the one used by Keskin and colleagues, consisted of a personalized neoantigen vaccine. The other vaccine consisted of non-mutated protein fragments corresponding to tumour-associated proteins present on cancer cells. This latter vaccine was not personalized to match each individual's proteins - the tumour-associated proteins were 

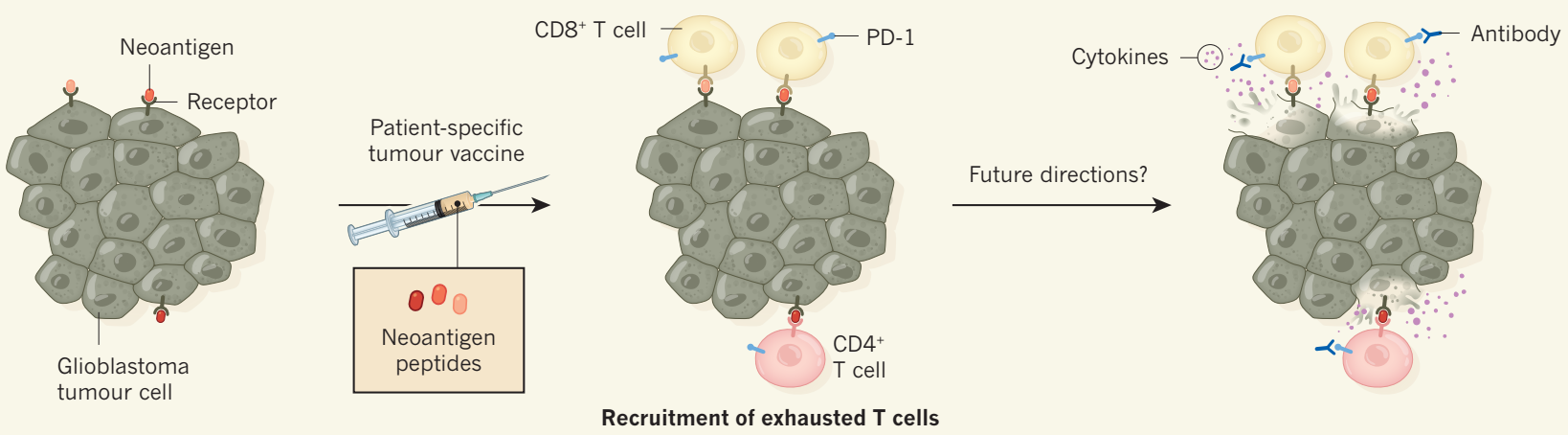

Figure 1 | Using immunotherapy to target human brain tumours. Keskin et al. ${ }^{4}$ and Hilf et al. ${ }^{5}$ report the outcomes of phase I clinical trials that tested ways of boosting immune responses against glioblastoma tumours, which are hard to target with immunotherapy. Both authors tried to enhance immune responses against tumour-specific, mutant versions of proteins, termed neoantigens, that are displayed by being bound to receptors on a person's tumour cells. Keskin et al. gave participants vaccines consisting of personalized sets of peptides (protein fragments) that matched the amino-acid sequences of their specific neoantigens. Hilf et al. combined such a personalized vaccine with one that targets non-mutant proteins common to this type of tumour (not shown). Both papers report that vaccination boosted immune responses involving $\mathrm{CD}^{+} \mathrm{T}$ cells and $\mathrm{CD}^{+} \mathrm{T}$ cells that recognized the neoantigens. However, this did not prevent cancer-associated death. Perhaps this is because T cells entered a dysfunctional state termed exhaustion, as reported by Keskin and colleagues. Entry into this state can be mediated by a T-cell-receptor protein called PD-1. Perhaps future studies will test whether blocking exhaustion by using antibodies that target PD-1 might enable such tumour-targeting T cells to remain active and secrete the cytokine molecules that aid tumour-cell destruction. identified through analysis of 30 glioblastomas, using techniques to identify proteins that are strongly associated with this type of tumour.

Of 15 people who had undergone surgery to remove their tumour and who were receiving chemotherapy, 4 received the non-personalized vaccine only and 11 received both vaccines, given consecutively. A couple of the people enrolled dropped out of the trial. A total of 13 people who had received the nonpersonalized vaccine generated an immune response that was evaluated, and 12 of these 13 individuals had $\mathrm{CD}^{+} \mathrm{T}$ cells that could recognize at least one protein in the non-personalized vaccine. The personalized vaccine induced $\mathrm{CD} 4^{+} \mathrm{T}$-cell responses targeting neoantigens in 8 out of 10 people who received the personalized vaccine and generated immune responses that were evaluated.

The two papers show that it is possible to make $\mathrm{T}$ cells that target some of the few neoantigens expressed by glioblastomas. Similar neoantigen-targeted vaccine approaches were previously tested ${ }^{6,7}$ in early-phase clinical trials for melanoma, which, unlike glioblastoma, is a type of cancer that has a high level of mutations and neoantigens.

These studies by Keskin, Hilf and their respective colleagues highlight several key aspects that underscore the current limitations of personalized-vaccine therapy, as well as the potential benefits. People with cancer could be receiving other treatments, which might limit the effectiveness of immunotherapy vaccines, as was observed by Keskin and colleagues for the participants who received steroids. By contrast, Hilf and colleagues did not report that T-cell responses were affected by the chemotherapy received by the people in their study. Although the sample sizes in these trials were small, the results suggest that the timing of vaccine administration should be carefully considered when designing future clinical trials in which multiple immunosuppressive agents are being used for treatment.

Several studies ${ }^{6-8}$ have shown that neoantigens are potent T-cell activators, and Hilf et al. demonstrate clearly that non-mutated proteins can also drive robust $\mathrm{CD} 8^{+} \mathrm{T}$-cell responses. This begs the question of which approach is superior for this type of therapy, or, indeed, whether combinations of mutated and nonmutated proteins might make the most effective vaccines, particularly for tumours that do not express many neoantigens.

As in vaccine studies in people with melanoma ${ }^{6,7}$, both glioblastoma trials preferentially elicited neoantigen-specific CD $4^{+}$ $\mathrm{T}$ cells, rather than $\mathrm{CD} 8^{+} \mathrm{T}$ cells. This was despite Keskin et al. selecting neoantigens that would be expected to trigger $\mathrm{CD} 8^{+} \mathrm{T}$ cells only. Interestingly, a strong $\mathrm{CD} 8^{+}$-T-cell response was raised only by the vaccine composed of non-mutated proteins. Future studies should explore the role of neoantigens and nonmutated proteins in eliciting cancer-killing $\mathrm{CD}^{+} \mathrm{T}$ cells, as well as the relative importance of responses of $\mathrm{CD} 4^{+} \mathrm{T}$ cells compared with $\mathrm{CD}^{+} \mathrm{T}$ cells to these proteins.

Although both studies demonstrated an immune-cell response that targeted tumours, most people who participated in the trials died from their cancer. Perhaps this was because the tumour-targeting T cells became exhausted, as suggested by the results of Keskin and colleagues. To get a more successful outcome in the future, combination therapies might be needed. For example, the administration of a vaccine along with checkpoint inhibitors might improve the quality of tumour-infiltrating T cells and tackle T-cell exhaustion. There is growing evidence that, to achieve the most effective immunotherapy results, it is necessary to enhance the number and quality of T cells infiltrating a tumour, ensuring the 'tuning up' of immune cells to effectively kill cancer cells ${ }^{9}$.

Combinatorial approaches might be particularly necessary in immunologically cold tumours ${ }^{8,10}$. A possible next step to build on the findings of Keskin, Hilf and their colleagues would be to conduct clinical trials of such combinatorial approaches. This approach has been effective in an animal model of pancreatic cancer ${ }^{8}$.

These two studies show that $\mathrm{T}$-cell responses can be boosted in cancers in which this was thought to be difficult. The findings suggest that further refinement of such approaches is a promising avenue of investigation in the search for therapies that target glioblastoma.

Neeha Zaidi and Elizabeth M. Jaffee are at the Sidney Kimmel Comprehensive Cancer Center, Johns Hopkins University School of Medicine, Baltimore, Maryland 21287, USA. e-mails:nzaidi1@jhmi.edu; ejaffee@jhmi.edu

1. Yarchoan, M. et al. N. Engl. J. Med. 377, 2500-2501 (2017).

2. Wolchok, J. D. et al. N. Engl. J. Med. 377, 1345-1356 (2017).

3. Yarchoan, M. et al. Nature Rev. Cancer 17, 209-222 (2017).

4. Keskin, D. B. et al. Nature 565, 234-239 (2019).

5. Hilf, N. et al. Nature 565, 240-245 (2019).

6. Ott, P. A. et al. Nature 547, 217-221 (2017).

7. Sahin, U. et al. Nature 547, 222-226 (2017).

8. Kinkead, H. L. et al. JCl Insight 3, e122857 (2018)

9. Friaetta, J. A. et al. Nature Med. 24, 563-571 (2018).

10.Popovic, A. et al. J. Clin. Invest. 128, 3209-3318 (2018).

E.M.J. declares competing financial and non-financial interests. See go.nature.com/2gacmz2 for details.

This article was published online on 19 December 2018. 